\title{
Die Einwirkung von Ammoniak auf Merkuronitrat.
}

\author{
Von
}

\section{Haridas SaHa und Kumud Nath Chotddurr. ${ }^{1}$}

Nach der Untersuchung über die Einwirkung von Ammoniak auf Merkurochlorid ${ }^{2}$ begannen wir die Reaktion zwischen Ammoniak und Merkuronitrat zu untersuchen. Wir fanden wie in dem früheren Falle, dal's bei Behandlung ron Merkuronitrat mit starkem Ammoniak (spez. Gew. 0.895) das ammoniakalische Filtrat beim Verdünnen mit Wasser einen weilsen und mit Schwefelwasserstoff einen schwarzen Niederschlag liefert, woraus hervorgeht, dafs ein Teil der Quecksilberverbindung in dem ammoniakalischen Filtrat vorbanden ist. Es wurde auch beobachtet, dafs in dem Glase, in welchem die Reaktion stattfand, feine Kügelchen von Quecksilber zurückblieben, die sich zu sehr grolsen Kugeln vereinigten, wenn das Glas eine Zeitlang in Dampfbade erwärmt wurde. Dies beweist direkt und sehr bündig, dals die schwarze Substanz aus Merkurosalzen und Ammoniak freies Quecksilber enthält. Bisher ist die Gegenwart von freiem Quecksilber indirekt durch Goldchlorid oder metallisches Gold bewiesen worden.

Das Quecksilber im ammoniakalischen Filtrat von Merkuronitrat wurde bestimmt, wobei man die Hälfte des im Merkuronitrat vorhandenen Quecksilbers fand. Bei einem Versuch enthielt das ammoniakalische Filtrat $36.8 \%$ Quecksilber rom Gewicht des angewandten Merkuronitrats, während in diesem theoretisch $71.42 \%$ vorhanden sind. Der geringe Unterschied war darauf zurückzuführen, dal's die benutzte Probe ron Merkuronitrat etwas Merkuridnitrat enthielt, obwohl das reinste Präparat von Merck verwendet wurde. Dies ergab sich daraus, dafs in dem Filtrat, das man bei Behandlung einer Probe mit Kochsalzlösung erhielt, Schwefelwasserstofi einen schwarzen Niederschlag lieferte. Das Merkuroquecksilber der Probe bestimmte man als $\mathrm{Hg}_{2} \mathrm{Cl}_{2}$, und zwar fand man $69.39 \%$ anstatt des theoretischen Wertes $71.42 \%$. Lis ist hier zu

1 Aus dem Manuskript ins Deutsehe übertragen von I. Koppel-Berlin.

${ }^{2}$ Z. anorg. Chem. 67 (1910), 357. 
erwähnen, dafs der weifse Niederschlag von Merkurinitrat und Ammoniak in starkem Ammoniak löslich ist. Diese Tatsache, sowie die in der früheren Mitteilung erwähnte zeigt deutlich, dafs BARFOED ${ }^{1}$ nicht berechtigt war, die Ansicht zu vertreten, dafs die schwarze Substanz aus Merkurosalzen und Ammoniak nur die Hälfte des Quecksilbers im freien Zustand enthalte. Hier sowohl wie beim Nerkurochlorid hängt die Menge des freien Quecksilbers in der schwarzen Substanz von der Konzentration des benutzten Ammoniaks und von der Art des Auswaschens ab; und in diesem besonderen Falle beim Merkuronitrat besteht der schwarze Niederschlag dieses Salzes mit überschüssigem Ammoniak vollständig aus freiem Quecksilber.

Das Filtrat von Merkuronitrat und überschïssigem Ammoniak wurde im Vakuum über konzentrierter Schwefelsäure eingedampft, wobei man ein weifses, nicht kristallisiertes Pulver erhielt. Man wusch es sorgfältig mit destilliertem Wasser und trocknete es über Calciumchlorid. Dies trockene Pulver erhitzte man dann im Dampfbad, wobei es aber keinen Gewichtsverlust erlitt. Beim Erhitzen im trockenen Reagenzglas zersetzte es sich plötzlich explosionsartig unter Entwickelung von roten nitrosen Dämpfen und Ammoniak; ein gelber Rückstand hinterblieb. Etwas Quecksilbersublimat wurde gleichfalls erhalten. Dies weifse Pulver scheint eine sehr stabile Verbindung zu sein, denn sie unterliegt nur sehr geringer Zersetzung bei mehrtägigem Kochen mit starker Kalilauge. Eine sorgfältige quantitative Analyse zeigte die folgende prozentische Zusammensetzung dieses weilsen Pulvers.

$\begin{array}{lcc} & 1 . & 2 . \\ \mathrm{Hg} & 78.31 \% & 78.09 \% \\ \mathrm{~N} & 5.82 & 5.75 \\ \mathrm{H} & 0.63 & 0.555 \\ \mathrm{O}(\text { durch Differenz) } & 15.24 & 15.61 \\ \left(\mathrm{NO}_{3}\right) & 11.82 & 12.28\end{array}$

Diese Zahlen entsprechen der empirischen Formel $\mathrm{N}_{2} \mathrm{Hg}_{2} \mathrm{H}_{4} \mathrm{O}_{5}$, aus der sich die folgenden theoretischen Werte berechnen:

$$
\begin{aligned}
\mathrm{Hg} & =78.12 \% \\
\mathrm{~N} & =5.46, \\
\mathrm{H} & =0.78, \\
\mathrm{O} & =15.62 \%, \\
\left(\mathrm{NO}_{3}\right) & =12.19,
\end{aligned}
$$

${ }^{1}$ Journ. Chem. Sore, Abstr. 1889. 
C. BARFOED, der zuerst die Einwirkung von Ammoniak auf Merkurosalze untersuchte, gab dem schwarzen Niederschlag aus Merkuronitrat und Ammoniak die Formel $3 \mathrm{Hg}+2\left(\mathrm{NH}_{2} \mathrm{HgNO}_{3}\right)$. $\mathrm{HgO} ;{ }^{1}$ für den zweiten Bestandteil dieses Gemisches berechnen sich die folgenden theoretischen Werte:

$$
\begin{aligned}
\mathrm{Hg} & =77.72 \% \\
\mathrm{~N} & =7.24, \\
\mathrm{H} & =0.51, \\
\mathrm{O} & =14.57, \\
\left(\mathrm{NO}_{3}\right) & =16.06 \%
\end{aligned}
$$

Die Mengen von Stickstoff, $\mathrm{NO}_{3}$ und Sauerstoff in dem zweiten Bestandteil dieses schwarzen Niederschlages - nach der Auffassung

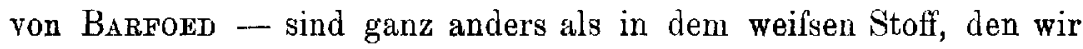
aus dem gleichen schwarzen Niederschlag durch starkes Ammoniak isolieren konnten. Es ist zu bemerken, dafs BARFoED, wenngleich er dem schwarzen Niederschlag die angegebene Zusammensetzung erteilte, doch den zweiten Bestandteil dieses Gemisches in reinem Zustand nicht isoliert zu haben scheint. Wir sind deswegen der Meinung, dafs er nicht ganz berechtigt war $z u$ behaupten, dafs der zweite Bestandteil dieses schwarzen Niederschlages aus Merkuronitrat $2\left(\mathrm{NH}_{2} \mathrm{Hg} . \mathrm{NO}_{3}\right) \mathrm{HgO}$ wäre.

\section{Experimentelles.}

Die verschiedenen Bestandteile des weilsen Pulvers aus dem ammoniakalischen Filtrat von Merkuronitrat und Ammoniak wurden folgenderwafsen bestimmt: Quecksilber bestimmte man als $\mathrm{HgS}$ in kalter salzsaurer Lüsung; $\mathrm{NO}_{3}$ durch Oxydation einer gegebenen Menge von reinem Eisendraht in Salzsäure und Zurücktitrieren des überschüssigen Ferroeisens mit 1/10-norm. Kaliumbichromatlösung; den Gesamtstickstoff ermitteite man nach Dumas und den Wasserstoff durch Verbrennung in einem Sauerstoffstrom, worauf man besondere Sorgfalt darauf verwendete, dals in das Chlorcalciumrohr kein Quecksilber hineinkam.

I. Vers. (1) $0.2154 \mathrm{~g}$ Substauz gaben $0.1957 \mathrm{~g} \mathrm{HgS.}$

(2) $0.1766 \mathrm{~g} \quad, \quad$ " $0.16 \mathrm{~g} \mathrm{HgS.}$

II. " (1) $0.2212 \mathrm{~g} \quad " \quad, 11.8 \mathrm{cem}$ feuchten stickstoff bei $26^{\circ}$ u. $748 \mathrm{~mm}$.

(2) $0.1892 \mathrm{~g} \quad " \quad$ " $10 \mathrm{ccm}$ feuchten Stickstoff bei $26^{\circ}$ u. $746 \mathrm{~mm}$.

1 Journ. Chem. Soc., Abstr. 1889; Journ. pralet. Chem. [2] 39201. 
III. Versuch (1) $0.4749 \mathrm{~g}$ Substanz gaben $0.0272 \mathrm{~g}$ Wasser.

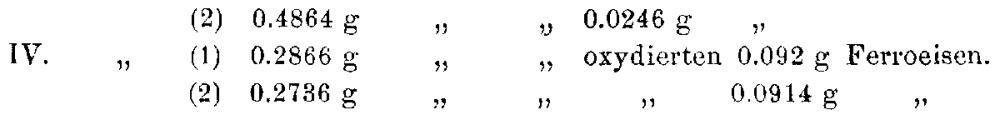

\section{Schlufsfolgerungen,}

Die beschriebene Untersuchung zeigt, dals die schwarze Substanz aus Merkuronitrat und Ammoniakflüssigkeit ein Gemisch von metallischem Quecksilber und einer weifsen Substanz der empirischen Formel $\mathrm{N}_{2} \mathrm{Hg}_{2} \mathrm{H}_{4} \mathrm{O}_{5}$ ist und dals demnach die Formel $2\left(\mathrm{NH}_{2} \mathrm{Hg}\right.$. $\left.\mathrm{NO}_{3}\right) \mathrm{HgO}$, die BABEOED dem zweiten Bestandteil des schwarzen Niederschlages beigelegt hat, nicht richtig ist. Überdies kann del schwarze Niederschlag nicht immer die Hälfte seines Quecksilbers in freiem Zustand enthalten, wie derselbe Forscher annimmt, da diese zweite Komponente in starkem Ammoniak reichlich löslich ist.

Eastern Bengal und Assam, Indien, Chemical Laboratiry, Dacca College.

Bei der Redaktion eingegangen am 15. Mai 1911. 